\title{
The Statistical Properties of Local Log-Contrast in Natural Images
}

\author{
Jussi T. Lindgren, Jarmo Hurri, and Aapo Hyvärinen \\ Helsinki Insititute for Information Technology \\ Department of Computer Science \\ University of Helsinki \\ firstname.lastname@cs.helsinki.fi
}

\begin{abstract}
The study of natural image statistics considers the statistical properties of large collections of images from natural scenes, and has applications in image processing, computer vision, and visual computational neuroscience. In the past, a major focus in the field of natural image statistics have been the statistics of outputs of linear filters. Recently, attention has been turning to nonlinear models. The contribution of this paper is the empirical analysis of the statistical properties of a central nonlinear property of natural scenes: the local log-contrast. To this end, we have studied both second-order and higher-order statistics of local log-contrast. Second-order statistics can be observed from the average amplitude spectrum. To examine higher-order statistics, we applied a higher-order-statistics-based model called independent component analysis to images of local log-contrast. Our results on second-order statistics show that the local log-contrast has a power-law-like average amplitude spectrum, similarly as the original luminance data. As for the higherorder statistics, we show that they can be utilized to learn intuitively meaningful spatial local-contrast patterns, such as contrast edges and bars. In addition to shedding light on the fundamental statistical properties of natural images, our results have important consequences for the analysis and design of multilayer statistical models of natural image data. In particular, our results show that in the case of local log-contrast, oriented and localized second-layer linear operators can be learned from the higher-order statistics of the nonlinearly mapped output of the first layer.
\end{abstract}

\section{Introduction}

The study of natural image statistics considers the statistical properties of large collections of images and their transformations (for a review see [1]). The most important application areas of natural image statistics are image processing and computer vision (e.g., [2]), and visual computational neuroscience (e.g., 34 ). In the case of image transformations, a majority of the research on natural image statistics has focused on the statistical properties of outputs of linear filters (e.g., 35.5). Recently, attention has been shifting to nonlinear models to account for higher-order visual information such as contours [6] and cue invariance [7]. 
One important nonlinear property of images is local luminance contrast, which in general refers to the relationship between the luminance of an object and its immediate surrounding. (From here on, the term 'local contrast' refers to local luminance contrast.) Spatial variations in local contrast can be viewed as a second-layen 11 visuel cue, because a model with two layers of filtering - with a nonlinearity in between - can be used to detect lines and edges formed by such cues (see, e.g., [8]). Such models are called filter-rectify-filter (FRF) models [8], where rectification refers to the nonlinear layer.

The contribution of this paper is an empirical study of the statistical properties of local contrast in natural images. While second-layer cues have received some attention in the natural image statistics research (e.g., 910; see also 7]), to our knowledge the statistical properties of local contrast have not been studied in detail in previous research. The importance of the study of the statistics of local contrast has further increased due to recent research, which suggests that local luminance and local contrast have small statistical dependencies in natural scenes [10]; this suggests the applicability of a particularly practical and simple (factorizable) statistical model, in which the cues are first modeled separately and then combined in a straightforward manner.

Here we have analyzed both the second-order (correlation-based) statistics of local contrast, and some higher-order statistics. Second-order statistics are revealed by the average amplitude spectrum. While the spectra of rectified signals have been derived in some special cases (e.g., [1]), the richness of image data makes it unfeasible to derive an analytical expression for filtered and rectified images. This intractability also holds for higher-order statistics, such as higherorder cumulants. Therefore, we have approached the problem by analyzing these statistics empirically in an ensemble of images.

The results of our empirical analysis suggest that in natural images, local log-contrast has important statistical properties. Here 'log' refers to the use of a logarithmic function as a nonnegative, compressive function in the computation of the contrast; in our model, this nonlinearity is closely related to the rectification in an FRF model (see below). Our results on amplitude spectra show that local log-contrast has similar second-order statistics as luminance: the average amplitude spectra of both fall of as $f^{-a}$, with $a>0$. This suggests that local log-contrast is scale invariant [12. As for the higher-order statistics, in this work they were probed with independent component analysis (or, equivalently, sparse coding), which has been a very influential higherorder-statistics-based method in the analysis of statistical properties of linear transformations of natural images 35. In the analysis of higher-order statistics, we were particularly interested in what kind of spatial patterns can be learned, because these patterns may form the second layer in FRF models. Our results show that meaningful spatial patterns of local contrast - such as contrast edges and bars - do emerge from higher-order statistics. This suggests that higher-order-statistics-based methods are able to discover

\footnotetext{
${ }^{1}$ A commonly used term for these cues is second-order, but we will use the term
} second-layer here to avoid confusion with second-order (correlation-based) statistics. 


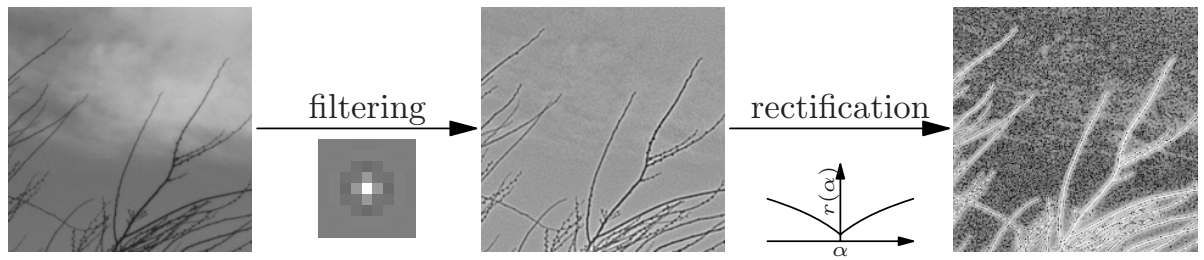

Fig. 1. The measure of local contrast employed in this paper is computed by a cascade of linear filtering and a pointwise logarithmic nonlinearity. The linear filter is a center-surround filter, and has been derived from the statistics of natural images (see text for details). This filter responds strongly (either positively or negatively) when the luminance difference between the center location and its surrounding is large, that is, when there is intuitively a large luminance contrast at the center location. The pointwise nonlinear logarithmic function (the rectification function) is $r(\alpha)=\ln (|\alpha|+d)$, $d \geq 1$, whose output is a nonnegative measure of local log-contrast; this function also compresses the large range of contrasts present in natural images. Note that in this figure, the images and the filter are represented with a highly different scale: the example images are of size $200 \times 200$, while the size of the filter is $9 \times 9$ pixels.

nonlinear image properties by learning second-layer linear operators in an FRF cascade.

The rest of this paper is organized as follows. In Section 2 we describe the measure of local contrast employed in this work. The natural image data is described shortly in Section 3. Second-order statistics are reported in Section 4. and results on higher-order statistics in Section 5. Finally, this paper ends with conclusions in Section 6 .

\section{Measuring Local Contrast}

Luminance contrast refers in general to the relationship between the luminance of an object and its immediate surrounding. There is no unique definition of contrast in a natural image [13. Two commonly employed types of measures of local luminance contrast utilize either center-surround filtering followed by a rectifying (nonnegative) nonlinearity, or localized measures of variance, possibly normalized by local luminance (see, e.g., [131410]). While there are many different ways of computing local contrast, in this paper we have chosen to apply the center-surround filtering approach 1315. (illustrated in Figure 1) due to its simplicity and relationship with other research on natural image statistics: it offers us a chance to specify the linear part of the computation of the local contrast - including the exact degree of localization - in terms of natural image statistics. This approach also gives us a view of the effect of the nonlinear part in the computation of contrast.

To specify the exact structure of the center-surround filter, we utilize a wellknown statistical criterion (e.g., [1617]): we specify that the filter will whiten the data - that is, the output of the filter will on the average have a flat power 
A

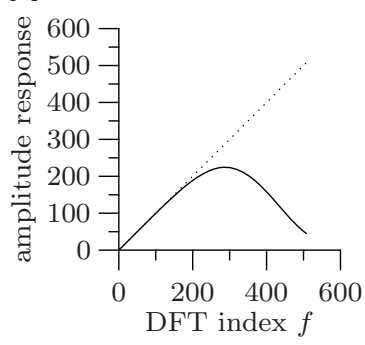

B

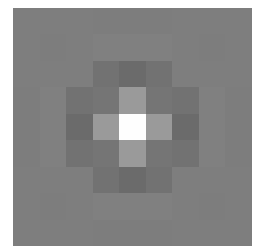

Fig. 2. The center-surround filter. (A) The specification of the filter in the Fourier domain. The amplitude response of the filter follows the linear whitening filter at low frequencies, but drops at high frequencies in order to not amplify high-frequency noise 17. For comparions, the plot also shows the amplitude response of the whitening filter with no noise control (dashed). The amplitude responses are plotted as functions of discrete Fourier transform (DFT) index. In the whitening filter, the cutoff frequency $f_{0}=0.4 N=407.2$, where $N=1018$ is the size of the images. (B) The central part of the resulting filter in the spatial domain.

spectrum - with the constraint that the filter should not amplify unduly the low-power high-frequency part of the spectrum, because a significant part of the highest frequencies consist of noise and sampling artifacts (see below). Because the average power spectrum is also the Fourier transform of the autocorrelation, the whitening condition is equivalent to spatial uncorrelatedness of filter output. The whitening criterion leads to the specification of the filter in the Fourier domain, where whitening determines the amplitude response of the filter, and the symmetrical structure of the filter results from setting the phase response to zero for all frequencies.

It is well-known that when averaged over a set of natural images, the amplitude spectrum falls off essentially as $f^{-a}$, where $f$ is the frequency, and $a>0$ and is typically between 0.7 and 1.5 (see, e.g., [14]). Therefore, for natural images, a whitening filter should have an amplitude response proportional to $f^{a}$; typically, the "average" value $a=1$ is used in the specification of a whitening filter. However, in whitening a precaution should be taken to avoid amplifying high-frequency noise and sampling artifacts [1617]. Because of the $f^{-a}$ amplitude spectrum of natural images, the high frequencies would need to be strongly amplified in whitening. Unfortunately this would magnify the high-frequency noise and sampling artifacts resulting from the use of a rectangular sampling grid. Therefore, a typical whitening filter drops off at high frequencies. Here we specify the exact form of the whitening filter as in [17]. Let $|G(f)|$ be the onedimensional Fourier amplitude response of the (spherically symmetric) filter $g$; then

$$
|G(f)|=f e^{-\left(f / f_{0}\right)^{4}},
$$

where $f_{0}$ is the frequency cut-off; here $f_{0}=0.4 N$, when $N \times N$ is the size of our natural images. The amplitude response of the filter is plotted in Figure2 A. The 
filter has the effect of removing the DC (constant component), dampening the low frequencies that are dominant in natural images, and attenuating the highest frequencies which have the worst signal-to-noise ratio. The two-dimensional whitening filter is a spherically symmetric $2 \mathrm{D}$ version of equation (1); Figure $2 \mathrm{~B}$ shows the resulting filter in the spatial domain. For details on the used filter, see [17. To improve computational efficiency, convolution with the filter was implemented in this work by pointwise multiplication in the Fourier space.

After filtering with the center-surround filter, the value of local contrast was obtained by applying a rectifying (nonnegative, nonlinear) function $r(\cdot)$ to the filtering output. Let $*$ denote convolution, $g(x, y)$ the center-surround filter, and $I(x, y)$ an $N \times N$ image; then the local contrast $c(x, y)$ is obtained by

$$
c(x, y)=r(g * I) .
$$

The exact form of the rectifying function $r(\cdot)$ is related to contrast gain control a way of compressing the large range of contrasts in images - which is a general property of the biological visual system [18] and is also used in image processing to facilitate the interpretation of images with high contrast (e.g., [19]). In this paper we examine in particular the local log-contrast, that is, the case where

$$
r(\alpha)=\ln (|\alpha|+d)
$$

where a relatively small constant $d$ is added to the absolute value to make the rectification function nonnegative and well-behaved (here $d$ is $10 \%$ of the mean of the absolute values of the center-surround-filtered images) . In psychophysics, the logarithmic nonlinearity is related to the relationship between stimulus contrast and the perceived contrast (e.g., 20]).

\section{$3 \quad$ Natural Image Data}

In the experiments we used the natural image dataset provided by van Hateren and van der Schaaf 4. This dataset contains over 4000 grayscale images of natural scenes, each image having a resolution of $1024 \times 1536$ pixels. Out of the two versions of these images available, we used the deblurred versions that have been compensated for the point spread function of the camera lens. To avoid border artifacts present in the data and to be able to apply the (square) whitening filter, we cropped each image to a resolution $1018 \times 1018$ pixels.

\section{Second-Order Statistics}

We examined the second-order statistics of the (original) luminance images, center-surround-filtered (whitened) images and local-log-contrast images. For this purpose, 200 natural images were sampled at random from the natural image collection, and the local-log-contrast computation scheme described in 


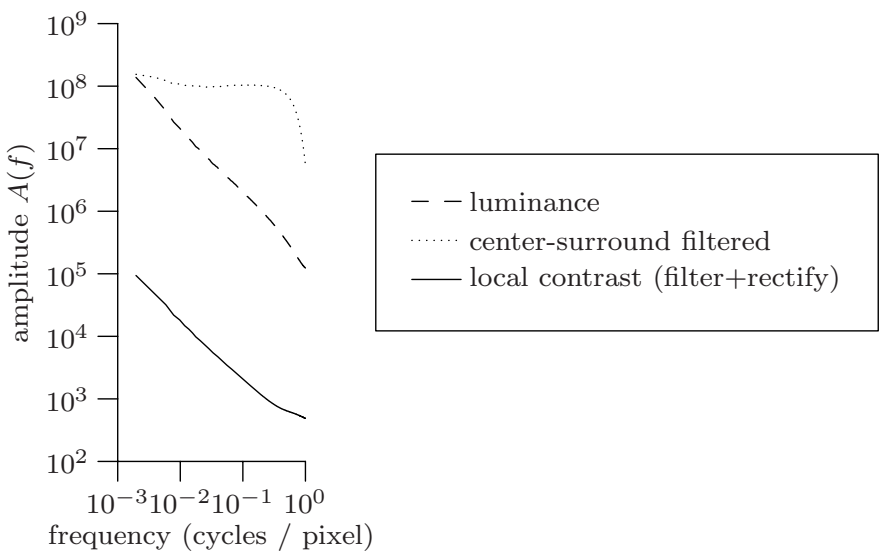

Fig. 3. Local log-contrast has similar second-order statistics as luminance: the average amplitude spectrum of local-log-contrast images exhibits similar $f^{-a}$ behaviour as the average amplitude spectrum of the luminance images. This log-log plot shows the amplitude spectra of luminance images, filtered images, and local-log-contrast images (filtering + rectification), averaged over 200 natural images and all orientations. In a log-log plot, a $f^{-a}$ characteristic corresponds to an affine plot with slope $-a$; the intercept reflects a scaling of the data and is irrelevant. Least-squares estimation of the slope and the intercept gives a slope $a_{\mathrm{lum}} \approx 1.2$ for the luminance data and $a_{\mathrm{con}} \approx 0.72$ for the local contrast data. Note the role of the rectification: before the rectification, the average amplitude spectrum of the filtered images is approximately flat (except for the highest frequencies), a consequence of the whitening principle.

Section 2 was applied to these images. Amplitude spectra averaged over the 200 images and all orientations were computed from the luminance images, centersurround-filtered images and local-log-contrast images.

The results are shown in Figure 3. please note that this is a log-log plot. As can be seen, the average amplitude spectrum of the luminance images follows the familiar $f^{-a}$ curve, which corresponds to an affine curve in a log-log plot; leastsquares estimation of an affine model Est $\left\{\log _{10} A_{\operatorname{lum}}\right\}(f)=b_{\operatorname{lum}}-a_{\operatorname{lum}} \log _{10} f$, where Est $\{\cdot\}$ denotes an estimator, gives a slope $a_{\text {lum }} \approx 1.2$. The intercept $b_{\text {lum }}$ corresponds to a global scaling of the data and is irrelevant here. The amplitude spectra of the center-surround images are approximately flat, except for the highest frequencies, as can be expected from the construction of the centersurround filter from the whitening principle (see Section 2).

The local contrast has a similar power-law-like $f^{-a}$ form as the luminance; least-squares estimation of an affine model yields $a_{\text {con }} \approx 0.72$ for the local contrast image data. Note in particular the effect of the rectifying nonlinearity, evident in the difference between the amplitude spectra of the center-surroundfiltered images and the rectified contrast images. This implies that in natural images, local log-contrast enjoys similar scale-invariance as luminance [12]. 


\section{$5 \quad$ Higher-Order Statistics}

In this work, the higher-order statistics were probed with independent component analysis (ICA), which has been an influential model in the analysis of higher-order statistics of natural images 445] and is closely related [17/5 to another influential model called sparse coding 3. In particular, we were interested in what kind of local-log-contrast patterns would emerge from higher-order statistics; it is well known that the luminance patterns that emerge from the application of ICA or sparse coding to luminance data are localized, oriented and bandpass and resemble - depending on the viewpoint - Gabor functions, edge and line detectors, and the receptive fields of simple cells in the primary visual cortex 174 .

In its basic form, ICA assumes that the observed data $\boldsymbol{x}$, which is a random vector of dimension $n$, has been generated by a linear generative model

$$
\boldsymbol{x}=\boldsymbol{A s},
$$

where $\boldsymbol{A}$ is a constant mixing matrix (to be estimated) and $s$ a random vector of unknown independent components. The dimension of $s$ is assumed to be equal to the dimension of $\boldsymbol{x}$, possibly after the observed data $\boldsymbol{x}$ have been reduced to a smaller dimension by principal component analysis [5. ICA tries to estimate both $\boldsymbol{s}$ and the parameter matrix $\boldsymbol{A}$ from the observed data $\boldsymbol{x}$; when the model holds, this can be done with very few assumptions (up to a scaling and ordering of the independent components and columns of matrix $\boldsymbol{A}$ ). In the absence of data following the generative model (4), many ICA algorithms - including the FastICA algorithm we use [5] - can still be interpreted as minimization of higher-order statistical dependencies between the components of the estimated random vector $\hat{\boldsymbol{s}}=\hat{\boldsymbol{A}}^{-1} \boldsymbol{x}[5$. In addition, when statistical dependencies are reduced by searching for maximally non-Gaussian projections - as in FastICA the projections often turn out to be sparse, and the method can be viewed as computing a sparse coding basis for the observed data.

We computed the ICA decomposition of localized local-log-contrast data; to be more precise, of local-log-contrast image patches of size $19 \times 19$. To compute this decomposition, we sampled a set of 100,000 patches of size $19 \times 19$ pixels from local-log-contrast images. Samples were taken from all of the over 4000 images. After sampling, the local DC component (the mean value in the patch, typically considered an "uninteresting" projection direction) was removed from each image patch. The patches were then vectorized; the resulting vectors formed a sample of the observed data $\boldsymbol{x}$ used in the ICA estimation. We used the FastICA algorithm [5] with $\tanh (\cdot)$ nonlinearity and symmetric (simultaneous) estimation of all components. The input dimension was $19 \times 19=361$, and principal component analysis was used to reduce the dimension for ICA to $16 \times 16=256$, retaining over $85 \%$ of the variance (dimensionality reduction is a standard procedure in ICA to reduce the effect of noise). This resulted in the estimation of 256 independent components, or maximally non-Gaussian directions.

The resulting set of ICA basis vectors (columns of matrix $\boldsymbol{A}$ in equation (4)) is shown in Figure 4A. As can be seen, these basis vectors of local-log-contrast 


\section{A}

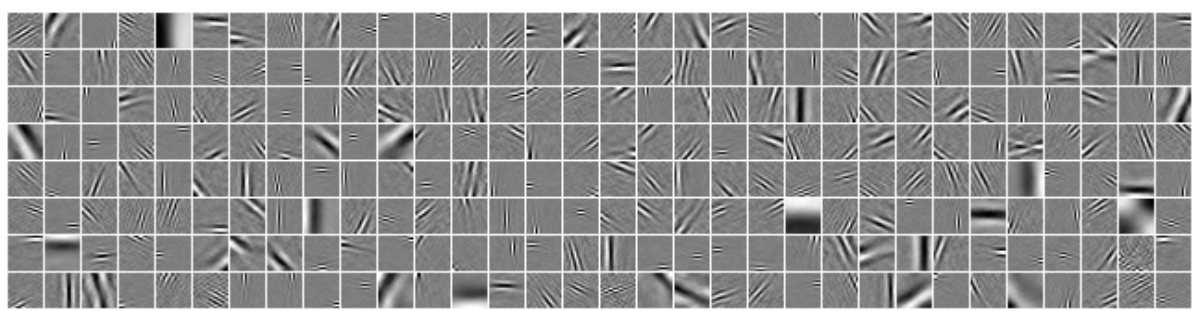

B

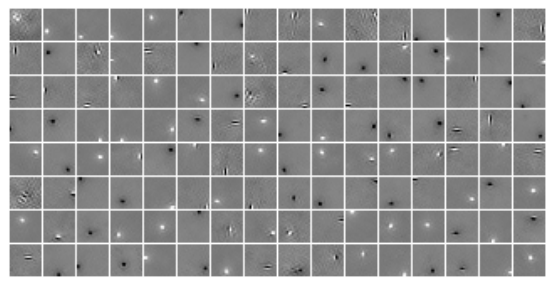

C

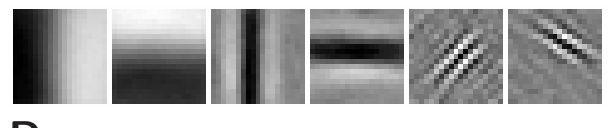

D

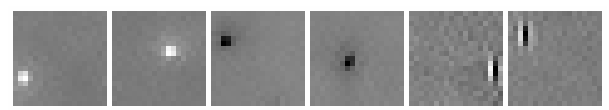

Fig. 4. Important contrast patterns emerge when independent component analysis (ICA) is applied to local-log-contrast data. (A) A set of 256 ICA basis vectors of size $19 \times 19$ pixels, computed from the local-log-contrast data. (B) For comparison, the ICA basis vectors obtained in a control experiment with a noncompressive rectification function $r(\alpha)=|\alpha|$. The basis vectors in (B) are the same pixel size as in (A), but are shown at a smaller scale. (C) Magnified examples of different types of locallog-contrast patterns in the basis vector set (A): contrast edges, contrast bars, and high-frequency localized spatial contrast patterns. (D) Magnified examples of different types of patterns from the control experiment results in (B).

data correspond to oriented spatial contrast patterns in many different scales (frequencies). These patterns correspond to spatial variations in contrast such as contrast edges, contrast bars and spatially localized frequency patterns; examples of these are shown in Figure 4C. These basis vectors could form the starting point of a second-layer statistical image representation. To our knowledge, learning such second-layer (FRF) representations of local contrast has not been demostrated in previous research. Our results are therefore an important step in the development of multilayer statistical models of natural image data. For example, when learning multilayer models of images, one approach is to learn the layers one by one. Here we have demonstrated that meaningful second-layer patterns can be learned by employing ICA on top of a rectified first-layer output after the first-layer filter has been learned from the whitening principle.

It should be noted that in our model, nonlinear detectors of contrast edges are not simply linear luminance edge detectors with rectified outputs. For example, whereas a rectified linear edge detector would respond positively to an edge regardless of the polarity (sign) of the edge, it would not signal the existence of a contrast edge, because there is no luminance difference across a contrast edge.

To verify that the results shown in Figure 4A are not trivial or an artifact, we performed two control experiments. 
1. The purpose of this control experiment was to study the role of the rectification ofunction. We repeated the computation of the ICA basis, but this time used the rectification function $r(\alpha)=|\alpha|$. The results are shown in Figure $4 \mathrm{~B}$, with close-ups in Figure 4D. As can be seen, in this case only a minority of the patterns are oriented, and these are of a single scale (high frequency); this suggests that a linear, noncompressive measure of contrast does not possess the statistical properties enjoyed by local log-contrast, which are needed to learn oriented spatial patterns in multiple scales. This indicates that in FRF architectures, the formation of subsequent processing layers may need appropriate gain control mechanisms in order to be able to perceive certain structures in the incoming data as salient.

2. The objective of this control experiment was to verify that our results are not an artifact of our model, but do indeed reflect the statistical properties of natural images. Here we repeated the computation of the ICA basis for three different types of white (uncorrelated) noise, each with different marginal distributions: Gaussian, uniform, and the same as in the original image data. For all of these noise models, the ICA estimation failed, suggesting that the filtered and rectified noise had a Gaussian distribution [5]. Our main results do therefore reflect the statistical structure of natural images.

\section{Conclusions}

We have shown in this paper that local log-contrast has important statistical properties in natural images. An examination of the average amplitude spectrum - which is equivalent to an examination of the second-order statistics - reveals a power-law form of $f^{-a}$, implying scale invariance. The ICA basis vectors - which reflect higher-order statistics - correspond to spatial local-log-contrast patterns such as contrast edges and bars. This suggests that higher-order statistics can be employed in learning multilayer statistical models of natural images.

The current work presents the first steps in the examination of the statistical properties of local log-contrast in natural images. For a more complete understanding of multilayer contrast processing, further theoretical and empirical research is under way. In particular, the effects caused by the interaction of the input data, the first-layer linear filter, and the used rectification function must be understood in more detail. One possibility is that the optimal parameterizations of the used mechanisms depend strongly on the requirements of subsequent processing stages.

Acknowledgments. This work was supported by the IST Programme of the European Community, under the PASCAL Network of Excellence, IST-2002506778 (J.L., A.H.), by the FDK Center of Excellence of the Academy of Finland (J.L.), and by the Academy of Finland project \#116962 (J.H.). This publication only reflects the authors' views. 


\section{References}

1. Simoncelli, E.P.: Statistical modeling of photographic images. In: Bovik, A. (ed.) Handbook of Image \& Video Processing, 2nd edn. pp. 431-441. Academic Press, San Diego (2005)

2. Portilla, J., Strela, V., Wainwright, M.J., Simoncelli, E.P.: Image denoising using scale mixtures of Gaussians in the wavelet domain. IEEE Transactions on Image Processing 12(11), 1338-1351 (2003)

3. Olshausen, B.A., Field, D.: Emergence of simple-cell receptive field properties by learning a sparse code for natural images. Nature 381(6583), 607-609 (1996)

4. van Hateren, J.H., van der Schaaf, A.: Independent component filters of natural images compared with simple cells in primary visual cortex. Proceedings of the Royal Society of London B. 265(1394), 359-366 (1998)

5. Hyvärinen, A., Karhunen, J., Oja, E.: Independent Component Analysis. John Wiley \& Sons, Chichester (2001)

6. Hoyer, P.O., Hyvärinen, A.: A multi-layer sparse coding network learns contour coding from natural images. Vision Research 42(12), 1593-1605 (2002)

7. Hurri, J.: Learning cue-invariant visual responses. In: Weiss, Y., Schölkopf, B., Platt, J. (eds.) Advances in Neural Information Processing Systems, vol. 18, pp. 539-546. The MIT Press, Cambridge (2006)

8. Landy, M.S., Graham, N.: Visual perception of texture. In: Chalupa, L.M., Werner, J.S. (eds.) The Visual Neurosciences, vol. 2, pp. 1106-1118. MIT Press, Cambridge (2004)

9. Johnson, A.P., Baker Jr., C.: First- and second-order information in natural images: a filter-based approach to image statistics. Journal of the Optical Society of America A. 21(6), 913-925 (2004)

10. Frazor, R.A., Geisler, W.S.: Local luminance and contrast in natural images. Vision Research 46(10), 1585-1598 (2006)

11. Regan, M.P.: Half-wave linear rectification of a frequency modulated sinusoid. Applied Mathematics and Computation 79(2-3), 137-162 (1996)

12. Ruderman, D.L.: Origins of scaling in natural images. Vision Research 37, 33583398 (1997)

13. Tadmor, Y., Tolhurst, D.J.: Calculating the contrasts that retinal ganglion cells and LGN neurones encounter in natural scenes. Vision Research 40(22), 3145-3157 (2000)

14. Bex, P.J., Makous, W.: Spatial frequency, phase, and the contrast of natural images. Journal of the Optical Society of America A. 19(6), 1096-1106 (2002)

15. Itti, L., Koch, C., Niebur, E.: A model of saliency-based visual attention for rapid scene analysis. IEEE Transactions on Pattern Analysis and Machine Intelligence 20(11), 1254-1259 (1998)

16. Atick, J.J., Redlich, A.N.: What does the retina know about natural scenes? Neural Computation 4(2), 196-210 (1992)

17. Olshausen, B.A., Field, D.: Sparse coding with an overcomplete basis set: A strategy employed by V1? Vision Research 37(23), 3311-3325 (1997)

18. Schwartz, O., Simoncelli, E.P.: Natural signal statistics and sensory gain control. Nature Neuroscience 4(8), 819-825 (2001)

19. Tumblin, J., Hodgins, J.K., Guenter, B.K.: Two methods for display of high contrast images. ACM Transactions on Graphics 18(1), 56-94 (1999)

20. Fiorentini, A., Maffei, L.: Contrast perception and electrophysiological correlates. Journal of Physiology 231(1), 61-69 (1973) 